\title{
Gestión del conocimiento organizacional: las incapacidades de aprendizaje y su relación con los estilos de aprendizaje en la industria papelera, cartonera y de celulosa de México
} Organizational knowledge management: the disabilities of learning and its
relationship to the styles of learning in the paper, carton and cellulose industry
in Mexico

José Gerardo Ignacio Gómez Romero

Universidad Juárez del Estado de Durango

igomez@ujed.mx

Francisco Martín Villarreal Solís

Universidad Juárez del Estado de Durango

fmvillasol@ujed.mx

María Deyanira Villarreal Solís

Universidad Juárez del Estado de Durango

devisol2000@yahoo.com.mx

\section{Resumen}

Este trabajo realiza un diagnóstico en la industria de celulosa, cartón y papel en México sobre las incapacidades de aprendizaje organizacional y los estilos de aprendizaje dominantes, además explora la relación entre estas variables, según Yeung, Ulrich, Nason y Von Glinow (2000), y sus efectos en el aprendizaje organizacional.

El instrumento de medición empleado se basó en Yeung et al. (2000), adaptado por Gómez (2008), y las respuestas se analizaron con un modelo de regresión lineal con las siete incapacidades de aprendizaje como variables predictoras de los cuatro estilos de aprendizaje. Se encontró evidencia estadística de una relación entre las incapacidades de aprendizaje y los estilos de aprendizaje organizacional en la industria estudiada.

Palabras clave: incapacidades de aprendizaje; estilos de aprendizaje; aprendizaje organizacional. 


\section{Abstract}

This work makes a diagnosis in the industry of pulp, cardboard and paper in Mexico on the organizational learning disabilities and dominant learning styles, it also explores the relationship between these variables, according to Yeung, Ulrich, Nason and Von Glinow (2000), and its effects on organizational learning. The measurement instrument used was based on Yeung et al. (2000), adapted by Gómez (2008), and the responses were analyzed with a linear regression model with the seven learning disabilities as predictor variables of the four learning styles. Statistical evidence of a relationship between the learning disabilities and organizational learning in the industry studied styles were found.

Key words: learning disabilities, learning styles, organizational learning.

\section{Fecha recepción: Agosto $2014 \quad$ Fecha aceptación: Noviembre 2014}

\section{Introducción}

El objetivo del presente estudio es indagar la posible relación entre las incapacidades de aprendizaje y los estilos de aprendizaje organizacional en la industria papelera, cartonera y celulosa de la República Mexicana, así como determinar qué incapacidad se presenta con más intensidad, además de establecer cuál es el estilo de aprendizaje organizacional dominante en el sector mencionado.

La Cámara del Papel (2013), señala la importancia de este sector y asevera que la producción mundial de esta industria ascendió a 400 millones de toneladas en 1999. Contra todos los pronósticos y predicciones de disminución del consumo de papel debido al auge de la era electrónica, desde 1980 se ha observado un crecimiento de $2.5 \%$ anual sostenido en la tasa de producción.

\section{Planteamiento del problema}


La globalización es el fenómeno social, económico y político que indudablemente ha afectado más en los últimos tiempos a la dinámica, la interacción y la economía entre las naciones, ya que además de cambiar su forma de convivencia y comunicación, modificó su manera de hacer negocios (Gómez, 2008).

Además, Puerto (2010) afirma que la economía global impuesta por este fenómeno llamado globalización, presenta un horizonte de claroscuros para los individuos, las organizaciones, las instituciones, las empresas y las naciones, mismos que deben aprender a manejar si desean permanecer vigentes en sus ámbitos de competencia, de lo contrario caerán en un precipicio sin fondo, donde la expulsión de los mercados es el final no deseado.

Gómez (2008) sostiene que actualmente la empresa se enfrenta a una mezcla de todos estos factores, razón por la cual un número importante de ellas desaparecen del mercado al no cumplir con los requerimientos que el entorno volátil y caprichoso les impone; las empresas que logren adecuarse de mejor manera a las circunstancias y a las nuevas reglas que impone el entorno altamente competitivo, serán las únicas capaces de sobrevivir, tal como señala Toffler (1999).

Para Yeung, et al. (2000), el conocimiento es el nuevo factor de éxito de las organizaciones de una sociedad posmoderna; sostienen que quienes logren generarlo mejor podrán sobrevivir y triunfar.

Huntington (2001) afirma que como consecuencia de los constantes cambios, ahora estamos en un cambio de época, una transición de la época industrial a la época del conocimiento.

En esta nueva era de la información y del conocimiento, la particularidad que distingue a las organizaciones triunfadoras es que generan conocimiento de mejor manera. Para Choo (1999), el aprendizaje se produce cuando los colaboradores de las organizaciones reaccionan a los cambios que se producen en el entorno, cuando se dan cuenta de sus errores y los corrigen, o cuando reaccionan ante las cambiantes demandas o gustos de los clientes.

Pero cuando las organizaciones no son capaces de reaccionar ante los cambios y exigencias de un entorno volátil y cambiante, hipotecan su futuro, lo cual se produce frecuentemente cuando las organizaciones no aprovechan el potencial que tienen, es decir, cuando no se benefician de su capacidad de aprendizaje, porque se enfrentan a las incapacidades o barreras de aprendizaje que les impide aprovechar de mejor manera el potencial que tienen dentro de sí (Yeung et al., 2000).

\section{Objetivo, pregunta de investigación e hipótesis}


Objetivo general: Indagar la posible relación entre las incapacidades de aprendizaje y los estilos de aprendizaje organizacional en la industria papelera, cartonera y celulosa de la República Mexicana.

Objetivo particular: Determinar qué incapacidad se presenta con más intensidad en las empresas estudiadas.

Objetivo particular: Establecer cuál es el estilo de aprendizaje organizacional dominante en el sector empresarial estudiado.

Preguntas de Investigación:

¿Existe relación entre las incapacidades de aprendizaje y los estilos de aprendizaje organizacional en la industria papelera, cartonera y de celulosa de la República Mexicana?

¿En caso de presentarse, qué incapacidades se presentan en mayor medida y más significativamente en los diferentes estilos de aprendizaje en la industria papelera, cartonera y de celulosa de la República Mexicana?

¿Cuál estilo de aprendizaje organizacional es el dominante en el sector estudiado? Hipótesis:

H1: Existe una relación significativa entre las incapacidades de aprendizaje y los estilos de aprendizaje organizacional en la industria papelera, cartonera y de celulosa de la República Mexicana.

H2: La incapacidad de aprendizaje que se presenta con mayor intensidad es la ceguera en las empresas estudiadas.

H3: El estilo de aprendizaje organizacional que se presenta con mayor intensidad es la de mejora continua en las empresas estudiadas.

\section{Justificación}

La decisión de estudiar esta industria se tomó debido a la importancia creciente de este sector, puesto que en la actualidad el mercado del sector papelero y sus productos derivados es de \$11 500 millones de dólares anuales, que equivalen al $6.3 \%$ del PIB manufacturero y representan $3.3 \%$ del PIB industrial del país.

La industria papelera a nivel mundial produjo ventas por 339.1 millardos de dólares en 2010, y en el mismo año solamente en Latinoamérica el mercado creció 28.8 \% (Price Waterhouse Coopers, 2011).

En México, la Cámara del Papel agrupa a la mayoría de los productores nacionales más importantes de papel, cartón, corrugados y derivados, quienes producen $98 \%$ de la 
producción nacional de esta industria. Con 27 empresas agremiadas a esta institución, los datos registrados aseveran que dicha industria genera más de 64000 empleos directos y 235 000 indirectos a través de 58 plantas en 20 estados del país (Cámara del Papel, 2013).

De la Madrid (2011) sostiene que del total de ventas de este sector durante 2008, que ascendió a 3000 millones de dólares, $38 \%$ se produjo en América del Norte, 34 \% en Europa y 18\% en Asia. Únicamente $4 \%$ se originó en Latinoamérica y $6 \%$ en África y Oceanía.

\section{Literatura revisada}

En la actualidad, el aprendizaje se ha vuelto clave para que las organizaciones sobrevivan y se conviertan en instituciones que aprenden. Como plantean Yeung et al. (2000) y Hernández y Martí (2006), esto no es una panacea para todas las debilidades de la organización, pero sí garantiza que dé una mejor respuesta a su entorno.

El uso del conocimiento como la nueva fuente de producción de riqueza está cambiando por completo la estructura de la sociedad y, por consiguiente, de las mismas organizaciones. Valdés (2002) afirma que si antes el saber se aplicaba al ser, en la nueva era se aplica al hacer, y aquellas instituciones que no sean capaces de generarlo están condenadas a desaparecer. La creación de conocimiento es una característica que distingue a las organizaciones que aprenden, por lo que son llamadas inteligentes.

El conocimiento en la actualidad se destaca como un factor clave para obtener competitividad en las organizaciones, y Probst, Raub y Romhardt (2001) aconsejan que estas se orienten a la generación de nuevas habilidades, productos, ideas y procesos más eficaces. También aceptan que el desarrollo del conocimiento es un pilar que complementa la adquisición del conocimiento mismo.

Por su lado, Choo (1999), y Beazley, Boenisch y Harden (2003), coinciden al afirmar que el aprendizaje del ente social se produce cuando los empleados de la organización reaccionan a los cambios que se verifican en el medio ambiente, al detectar errores y corregirlos, así como al modificar estrategias, supuestos o normas, todo ello vital para la productividad, la innovación y el desempeño de las empresas y los empleados.

Yeung et al. (2000), aseveran que los individuos y las organizaciones poseen cuatro estilos para aprender:

1. La experimentación, donde se aprende probando ideas nuevas y adoptando una posición receptiva del entorno. 
2. La adquisición de competencias, donde las personas y los equipos adquieren competencias nuevas; se centra en la experiencia de terceros.

3. Las marcas de referencia (benchmarking), donde se aprende averiguando cómo funcionan otros para después tratar de adaptar dichos conocimientos.

4. Las mejoras continuas, donde se aprende mejorando constantemente lo que ya se hacía.

Estos estilos solamente representan tipos ideales de aprendizaje. Al igual que las personas que jamás se ciñen a un único tipo de temperamento cuando se desenvuelven en su vida cotidiana, aunque tengan un temperamento dominante-, las empresas y los individuos rara vez aprenden exclusivamente con un solo estilo. Yeung et al. (2000), señalan que las organizaciones no deben limitarse proponiendo uno solo de estos estilos, sino todo lo contrario, deben tratar de utilizar todos en alguna etapa de la vida.

Etkin (2007) y Gómez (2008) coinciden al afirmar que las organizaciones aprenden cuando las ideas y los conocimientos que generan sus empleados son compartidos más allá de los límites del espacio físico de la organización; sin embargo, también es preciso entender que el aprendizaje atraviesa por muchos pequeños fracasos y que algunos de ellos pueden hacer que la organización se dirija a su aniquilamiento, a menos que dichos fracasos sean inteligentes, es decir, que permitan aprender de ellos y fortalecerse para enfrentar la realidad futura.

Por otro lado, todas las organizaciones y los negocios generan en mayor o menor medida conocimiento organizacional, pero cuando se enfrentan a un entorno volátil, complejo y competitivo, como el actual, muchas terminan por fracasar y cerrar. En la era del conocimiento que vivimos, solo sobrevivirán aquellas organizaciones capaces de generar y aplicar el conocimiento organizacional (Yeung et al., 2000).

Dichos autores argumentan que no es casualidad que la mayoría de las organizaciones aprendan mal, y coinciden con Argyris (1993) cuando afirman que la incipiente formación administrativa de los gerentes y dueños de los negocios, acarrea necesariamente deficiencias en la generación del conocimiento organizacional (CO). A estas se les denomina barreras o incapacidades de aprendizaje.

El término barreras o incapacidades de aprendizaje se refiere a las condiciones que dificultan u obstaculizan el aprendizaje en el ámbito de las organizaciones y de los negocios y que impiden que la organización, independientemente del estilo de aprendizaje que esta utilice, genere conocimiento organizacional. Son como una enfermedad silenciosa: no dan síntomas de dolor; sin embargo, evitan que algunos procesos se realicen asertivamente y terminan por volver inoperante a la organización cuando la invaden por completo. 
Si dichas barreras no se identifican a tiempo y se superan, ciertamente las organizaciones seguirán generando aprendizaje organizacional, pero este será deficiente y estará plagado de deficiencias y errores, desde su generación hasta su generalización.

En general, los autores coinciden en los efectos que provocan las barreras, aunque algunos las desglosan más que otros. A continuación se presenta el modelo de Yeung et al. (2000), basado en 7 incapacidades:

1. Ceguera: Impide evaluar correctamente las amenazas y oportunidades del entorno, dejando pasar un sinfín de oportunidades.

2. Candidez: Se presenta cuando hay deficiencias en el análisis de las situaciones y generación de soluciones. Ante situaciones complejas se aplican las reglas conocidas sin hacer un análisis cuidadoso.

3. Homogeneidad: Solo toma en cuenta el punto de vista del dueño o gerente, quien es omnisciente y experto en todo.

4. Acoplamiento estrecho: La inflexibilidad y no adaptabilidad es la tónica, que se deriva del análisis cándido e incompleto de la realidad.

5. Parálisis: Impide tomar medidas o implantar procedimientos nuevos, no porque se desconozca la manera de realizarlos, sino porque se dedica demasiado tiempo al análisis.

6. Aprendizaje de supersticiones: Impide interpretar correctamente el significado de la experiencia, además de que culpa a terceras personas de todo lo malo que ocurre en el negocio, difundiendo ideas equivocadas de la situación.

7. Difusión deficiente: Se presenta cuando las personas aprenden cosas nuevas, pero no las difunden a otras personas y/o áreas del negocio y, en consecuencia, no surge el aprendizaje en grupo.

\section{Metodología}

El presente trabajo es correlacional puesto que, de acuerdo con Hernández, Fernández y Baptista (2006), busca establecer una relación entre las variables. Además, es transversal pues la toma de información se limitó a una sola ocasión, y también es no experimental, porque se obtiene y analiza la información sin modificar ninguna de las condiciones existentes.

El instrumento de medición que se aplicó fue utilizado por Yeung et al. (2000), en un estudio que realizaron en 300 empresas, probado y validado de acuerdo al entorno mexicano por Gómez (2008). Consta de 58 reactivos, con una escala tipo Likert de seis opciones, 
gradualmente en ascenso de la siguiente forma: "no sé/no aplica, muy poco, poco, algo, mucho y muchísimo".

Para determinar el universo se utilizó el directorio de la Cámara del Papel, que es la institución que agremia a los principales fabricantes de papel, de empaque y de cartón: conjunta a 27 socios, 2 agremiados y 3 cooperadores, que tienen presencia en 20 estados de México. Dentro de sus socios se encuentran firmas reconocidas como Kimberly-Clark de México, BioPappel, Procter \& Gamble, Copamex y Cartón Ponderosa, entre otras.

Se logró la colaboración de 25 empresas de las 32 agremiadas a la Cámara del Papel y que tienen presencia en 15 estados de México, con un total de 420 cuestionarios que contestaron directores, gerentes, jefes de departamentos y supervisores (mandos medios, gerenciales y directivos).

Los datos obtenidos se capturaron y se trabajaron en el programa estadístico SPSS versión 19.0, se calculó la confiabilidad del instrumento por medio de la prueba Alfa de Cronbach, cuyos resultados se pueden apreciar en la Tabla 1. Barraza-Macías (2008) sostiene que el valor del $\square \square$ de Cronbach menor a .60 es mediocre, de .60 a .65 es indeseable, de .65 a .70 es aceptable, de .70 a .80 es respetable y de .80 a .90 es muy buena. Esta prueba se corrió para cada dimensión (estilos e incapacidades).

Tabla 1: Confiabilidad del instrumento de medición

\begin{tabular}{|l|c|c|}
\hline Prueba utilizada & Dimensión & Valor obtenido \\
\hline Alfa de Cronbach & Estilos de Aprendizaje & 0.868 \\
\cline { 2 - 3 } & Incapacidades de Aprendizaje & 0.784 \\
\hline
\end{tabular}

Fuente: Elaboración propia.

\section{Resultados}

Para dar respuesta al objetivo de investigación y a las hipótesis planteadas se generaron las tablas 2 y 3 , que muestran los promedios y las frecuencias de las incapacidades de aprendizaje dominante y de los estilos de aprendizaje que se presentan con mayor frecuencia en el sector industrial estudiado, donde destacan la mejora continua como el estilo de aprendizaje más frecuentemente utilizado y la ceguera como la incapacidad más dominante.

Tabla 2: Estilos de aprendizaje. Promedios y frecuencia.

\begin{tabular}{|l|l|l|}
\hline Estilos de aprendizaje & Promedio & Frecuencia \\
\hline
\end{tabular}




\begin{tabular}{|l|c|c|} 
Mejora Continua & 4.1 & 183 \\
\hline Marcas de Referencia & 3.4 & 44 \\
\hline Experimentadoras & 3.9 & 150 \\
\hline Adquisición de Competencias & 3.4 & 45 \\
\hline
\end{tabular}

Fuente: Elaboración propia.

Tabla 3: Incapacidades de aprendizaje. Promedios y frecuencia.

\begin{tabular}{|l|c|c|}
\hline Incapacidades de aprendizaje & Promedio & Frecuencia \\
\hline Ceguera & 3.86 & 222 \\
\hline Candidez & 3.53 & 51 \\
\hline Homogeneidad & 2.89 & 13 \\
\hline Acoplamiento Estrecho & 2.98 & 20 \\
\hline Parálisis & 3.30 & 47 \\
\hline Aprendizaje de Supersticiones & 3.03 & 42 \\
\hline Difusión Deficiente & 3.46 & 27.00 \\
\hline
\end{tabular}

Fuente: Elaboración propia.

Para saber si existen diferencias estadísticamente significativas entre los grupos correspondientes a cada tipo de estilos de aprendizaje y de las incapacidades, se aplicó un análisis de la varianza con una prueba F, misma que de acuerdo con Lind, Marchal y Wathen (2005), se utiliza con el fin de probar si dos muestras provienen de poblaciones que tienen varianzas iguales (la cual se considera hipótesis nula). Los análisis se presentan en las tablas 4 y 5 , respectivamente.

En la primera columna se muestran los tipos de cultura, en la segunda se indican las fuentes de varianza, en la tercera se muestra la suma de los cuadrados ya calculados, en la cuarta columna se disponen los grados de libertad asociados a cada fuente de varianza, en la quinta columna se presenta el cuadrado de la media, en la sexta se presentan los resultados de la prueba $\mathrm{F}$ y, por último, en la séptima columna se presenta el nivel de significación que muestra el peso de la evidencia contra la hipótesis nula.

Tabla 4: Análisis de varianza con un factor. Estilos de aprendizaje.

\begin{tabular}{|l|l|l|l|l|l|l|}
\hline 1 & 2 & 3 & 4 & 5 & 6 & 7 \\
\hline
\end{tabular}




\begin{tabular}{|c|c|c|c|c|c|c|}
\hline & & $\begin{array}{l}\text { Suma de } \\
\text { cuadrados }\end{array}$ & gl & $\begin{array}{c}\text { Media } \\
\text { cuadrática }\end{array}$ & $F$ & Sig. \\
\hline \multirow[t]{3}{*}{ Mejora continua } & $\begin{array}{l}\text { Entre } \\
\text { grupos }\end{array}$ & 240.862 & 274 & .879 & 5.892 & .000 \\
\hline & $\begin{array}{l}\text { Intra } \\
\text { grupos }\end{array}$ & 20.142 & 135 & .149 & & \\
\hline & Total & 261.004 & 409 & & & \\
\hline \multirow[t]{3}{*}{$\begin{array}{l}\text { Marcas de } \\
\text { referencia }\end{array}$} & $\begin{array}{l}\text { Entre } \\
\text { grupos }\end{array}$ & 390.283 & 274 & 1.424 & 5.180 & .000 \\
\hline & $\begin{array}{l}\text { Intra } \\
\text { grupos }\end{array}$ & 37.125 & 135 & .275 & & \\
\hline & Total & 427.408 & 409 & & & \\
\hline \multirow[t]{3}{*}{ Experimentadoras } & $\begin{array}{l}\text { Entre } \\
\text { grupos }\end{array}$ & 393.605 & 274 & 1.437 & 6.229 & .000 \\
\hline & $\begin{array}{l}\text { Intra } \\
\text { grupos }\end{array}$ & 31.131 & 135 & .231 & & \\
\hline & Total & 424.736 & 409 & & & \\
\hline \multirow[t]{3}{*}{$\begin{array}{l}\text { Adquisición de } \\
\text { competencias }\end{array}$} & $\begin{array}{l}\text { Entre } \\
\text { grupos }\end{array}$ & 305.973 & 274 & 1.117 & 4.090 & .000 \\
\hline & $\begin{array}{l}\text { Intra } \\
\text { grupos }\end{array}$ & 36.857 & 135 & .273 & & \\
\hline & Total & 342.831 & 409 & & & \\
\hline
\end{tabular}

Fuente: Elaboración propia.

Tabla 5: Análisis de varianza con un factor. Incapacidades de aprendizaje.

\begin{tabular}{|c|c|c|c|c|c|c|}
\hline 1 & 2 & 3 & 4 & 5 & 6 & 7 \\
\hline & & $\begin{array}{l}\text { Suma de } \\
\text { cuadrados }\end{array}$ & $\mathrm{gl}$ & $\begin{array}{c}\text { Media } \\
\text { cuadrática }\end{array}$ & $\mathrm{F}$ & Sig. \\
\hline \multirow[t]{3}{*}{ Mejora continua } & $\begin{array}{l}\text { Entre } \\
\text { grupos }\end{array}$ & 240.862 & 274 & .879 & 5.892 & .000 \\
\hline & $\begin{array}{l}\text { Intra } \\
\text { grupos }\end{array}$ & 20.142 & 135 & .149 & & \\
\hline & Total & 261.004 & 409 & & & \\
\hline \multirow[t]{3}{*}{$\begin{array}{l}\text { Marcas de } \\
\text { referencia }\end{array}$} & $\begin{array}{l}\text { Entre } \\
\text { grupos }\end{array}$ & 390.283 & 274 & 1.424 & 5.180 & .000 \\
\hline & Intragrupos & 37.125 & 135 & .275 & & \\
\hline & Total & 427.408 & 409 & & & \\
\hline \multirow[t]{3}{*}{ Experimentadoras } & $\begin{array}{l}\text { Entre } \\
\text { grupos }\end{array}$ & 393.605 & 274 & 1.437 & 6.229 & .000 \\
\hline & $\begin{array}{l}\text { Intra } \\
\text { grupos }\end{array}$ & 31.131 & 135 & .231 & & \\
\hline & Total & 424.736 & 409 & & & \\
\hline \multirow[t]{3}{*}{$\begin{array}{l}\text { Adquisición de } \\
\text { competencias }\end{array}$} & $\begin{array}{l}\text { Entre } \\
\text { grupos }\end{array}$ & 305.973 & 274 & 1.117 & 4.090 & .000 \\
\hline & $\begin{array}{l}\text { Intra } \\
\text { grupos }\end{array}$ & 36.857 & 135 & .273 & & \\
\hline & Total & 342.831 & 409 & & & \\
\hline
\end{tabular}

Fuente: Elaboración propia. 
Como se puede observar, la diferencia de medias es significativa en todos los grupos, lo cual implica que están diferenciados de acuerdo con el procedimiento estadístico aplicado, por lo que los resultados mostrados anteriormente son confiables.

Con el propósito de contrastar las hipótesis de estudio, se aplicaron modelos de regresión lineal usando el procedimiento de mínimos cuadrados ordinarios para evaluar la relación lineal de dependencia, que de acuerdo con Hair, Anderson, Tatham y Black (2006), es con mucho la técnica de dependencia más utilizada y versátil, aplicable en cualquier ámbito de los negocios, pudiendo ser empleada para analizar la relación entre una variable criterio y una o varias variables independientes.

Cabe destacar que los modelos de regresión se aplicaron de la siguiente manera: se contrastaron cada uno de los cuatro tipos de estilos de aprendizaje con cada una de las siete incapacidades; las variables independientes fueron las incapacidades. Lo anterior generó un conjunto de 28 modelos (7 tipos de incapacidades por 4 estilos de aprendizaje). Dichos resultados se muestran en la tabla 6 .

Tabla 6: Resumen del modelo de regresión por variable

\begin{tabular}{|c|c|c|c|c|c|c|c|c|c|c|c|c|}
\hline \multirow{2}{*}{$\begin{array}{l}\text { Variable } \\
\text { Dependiente } \\
1\end{array}$} & \multicolumn{3}{|c|}{ Experimentadoras } & \multicolumn{3}{|c|}{$\begin{array}{l}\text { Marcas de } \\
\text { referencia }\end{array}$} & \multicolumn{3}{|c|}{$\begin{array}{l}\text { Adquisición de } \\
\text { competencias }\end{array}$} & \multicolumn{3}{|c|}{ Mejora continua } \\
\hline & 2 & 3 & 4 & 5 & 6 & 7 & 8 & 9 & 10 & 11 & 12 & 13 \\
\hline $\begin{array}{l}\text { Variable } \\
\text { Independiente }\end{array}$ & $r^{2}$ & B & Sig. & $r^{2}$ & $\mathrm{~B}$ & Sig. & $r^{2}$ & B & Sig. & $r^{2}$ & B & Sig \\
\hline Ceguera & 0.424 & 0.670 & 0.000 & 0.389 & 0.643 & 0.000 & 0.345 & 0.543 & 0.000 & 0.380 & .498 & 0.000 \\
\hline Candidez & 0.122 & 0.483 & 0.000 & 0.135 & 0.509 & 0.000 & 0.122 & 0.434 & .000 & 0.151 & 0.422 & .000 \\
\hline Homogeneidad & 0.024 & 0.166 & 0.002 & 0.062 & 0.265 & 0.000 & 0.024 & 0.147 & 0.002 & 0.014 & 0.100 & 0.015 \\
\hline $\begin{array}{l}\text { Acoplamiento } \\
\text { estrecho }\end{array}$ & \begin{tabular}{|c|}
- \\
0.002 \\
\end{tabular} & $\begin{array}{c}- \\
0.011\end{array}$ & 0.866 & 0.005 & 0.093 & 0.137 & -0.002 & -0.005 & 0.926 & 0.003 & -0.053 & 0.280 \\
\hline Parálisis & 0.000 & 0.024 & 0.746 & 0.012 & 0.162 & 0.030 & 0.001 & 0.039 & 0.564 & 0.005 & 0.082 & 0.165 \\
\hline $\begin{array}{l}\text { Aprendizaje } \\
\text { supersticiones }\end{array}$ & 0.026 & 0.169 & .001 & 0.002 & -0.049 & 0.344 & 0.019 & -0.138 & 0.003 & 0.024 & -0.129 & 0.002 \\
\hline $\begin{array}{l}\text { Difusión } \\
\text { deficiente }\end{array}$ & 0.148 & 0.542 & 0.000 & 0.139 & 0.527 & 0.000 & 0.177 & 0.532 & 0.000 & 0.172 & 0.458 & 0.000 \\
\hline
\end{tabular}

Fuente: Elaboración propia.

Se asume de acuerdo con la literatura revisada, que existe una relación entre las incapacidades de aprendizaje organizacional y los estilos de aprendizaje organizacional. Con base en lo anterior, este modelo incluye a la variable independiente "incapacidades de aprendizaje organizacional" como explicativa de los estilos de aprendizaje organizacional.

$$
Y_{\text {estilos de aprendizaje }}=a+b_{1} X_{\text {incapacidades de aprendizaje }}+€
$$


En donde:

$Y_{\text {Ede A }}=$ Estilos de aprendizaje organizacional

$\alpha=$ Intercepto

$\mathrm{X}_{\text {Inc. de ap. }}$ Incapacidades de aprendizaje organizacional

$\epsilon=$ Término de error

$\mathrm{b}_{1},=$ Coeficiente asociado a la variable independiente

Con los siguientes resultados:

$$
Y_{E \text { de A }}=1.999+.541 X \text { Inc. de ap. }
$$

(t) $\quad(8.816)(8.245)$

$$
R^{2}=.143
$$

Resulta estadísticamente significativo el impacto de la variable independiente "Incapacidades de aprendizaje organizacional", sobre los estilos de aprendizaje organizacional. Es importante resaltar que los objetivos del análisis se cumplen cabalmente al evaluar la significancia estadística de la variable independiente sobre la variable dependiente con el estadístico t de student y su p-valor asociado de .000 .

\section{Conclusiones}

Con base en los resultados estadísticos obtenidos, podemos afirmar que estadísticamente existe evidencia de una relación significativa entre las incapacidades de aprendizaje y los estilos de aprendizaje organizacional, confirmando lo expresado en la literatura analizada.

Argyris (1999) y Gómez, Villarreal y Villarreal (2014) coinciden al afirmar que todas las organizaciones y los negocios generan en mayor o menor medida conocimiento organizacional, estén conscientes o lo ignoren, estén preparadas para ello o no, y que son las incapacidades las que impiden que el conocimiento se genere y se generalice de mejor manera.

De acuerdo a los resultados obtenidos, podemos afirmar que las incapacidades de aprendizaje influyen en los estilos de aprendizaje organizacional que las empresas usan para resolver sus problemas y dar respuesta a sus clientes.

Gómez (2008), afirma que no se debe de olvidar que la presencia de una sola incapacidad puede ser suficiente para disminuir e incluso bloquear el aprendizaje en una organización, y que las incapacidades son como enfermedades silenciosas que invaden a la organización. Sus dueños o gerentes no se dan cuenta de lo que sucede, y cuando reparan en su presencia es demasiado tarde, 
pues la organización generalmente ya se encuentra en gran medida infectada y el estilo de aprendizaje resulta afectado.

A pesar de que el sector estudiado es por naturaleza altamente competitivo, y en consecuencia los directores y gerentes de las diferentes plantas son académica y técnicamente competentes, temas como las incapacidades de aprendizaje y la manera como estas afectan los estilos de aprendizaje de las empresas, son generalmente ajenos a su bagaje cultural y, por lo tanto, desconocidos para ellos. En la práctica pueden ver sus efectos y consecuencias, que terminan por volver ineficientes a sus empresas y a veces llevarlas al cierre, pero no logran vislumbrar qué es lo que provoca las ineficiencias en los procesos productivos o administrativos de sus fábricas.

Aquí vale la pena reflexionar acerca de la forma como aprenden las empresas, ya que depende en primer término de cómo descubren problemas, y de cómo inventan soluciones para mantenerse en el negocio. Asimismo, es necesario que los líderes de las empresas estén enterados de estos temas vitales para el desarrollo de cualquier negocio, sobre todo si se desenvuelven en un ambiente competitivo, como es el caso que nos ocupa en el presente estudio.

Por último, la respuesta al segundo de los objetivos de investigación y su correspondiente pregunta de investigación e hipótesis, es: Es preciso definir el estilo de aprendizaje organizacional que se presenta con más intensidad en las empresas estudiadas. A partir de los cálculos estadísticos, se puede concluir que la mejora continua es el estilo de aprendizaje dominante en la industria papelera, cartonera y de celulosa en México. Esto habla de una manera particular de realizar el trabajo cotidiano por parte de la industria estudiada, que estriba en dominar una forma de hacer las cosas; hasta que se tiene control sobre todas las variables de producción y comercialización, se inicia un proceso gradual de mejora en cada una de las actividades que se realizan.

La paradoja consiste en que el estilo de aprendizaje dominante en la industria estudiada es propio de empresas que enfrentan mercados estables, sin cambios significativos y con una competencia moderada; sin embargo, las características de los mercados a los que se enfrentan dichas empresas son de volatilidad y alta competitividad.

Tal vez la explicación a lo anterior sea que este sector industrial generalmente está sujeto a un sinnúmero de reglamentaciones fiscales, financieras, contables y ambientales, que lo obligan a tomar decisiones utilizando el tacto, midiendo el riesgo en cada cambio que desea hacer.

La respuesta al tercer objetivo de investigación y su correspondiente hipótesis y pregunta de investigación, es: Determinar la incapacidad que se presenta con mayor intensidad en las empresas estudiadas. Como resultado de los cálculos estadísticos obtenidos, se puede afirmar que la ceguera es la incapacidad que se presenta con mayor frecuencia, y que generalmente lo hace cuando la empresa está ensimismada, preocupada por sobrevivir. De esa manera, termina por padecer 
ceguera de taller, es decir, sus problemas absorben en demasía su energía y atención, distrayéndola de la volatilidad del entorno, un factor determinante para que los negocios lleguen a cerrar o no obtengan los resultados pronosticados.

Al parecer, lo anterior no es propio de las empresas estudiadas, pero es bueno tomar en cuenta que la ceguera acompañada de la candidez (segunda incapacidad en intensidad), lleva a los responsables de las empresas a dejar de evaluar todos los alcances de la competencia y del entorno donde se desenvuelven, poniendo en riesgo su presencia en el mercado.

Al llegar a este punto debemos enfatizar que la presencia de las incapacidades de aprendizaje en las organizaciones es un tema que debe preocupar a los dueños, gerentes y estudiosos del aprendizaje organizacional, ya que impiden que el conocimiento se genere de mejor manera en las empresas. Lamentablemente, este tema no está ligado al parecer de manera directa a temas tan sensibles como la productividad o la rentabilidad de las empresas y por ello pasa inadvertido por los responsables de las empresas, quienes terminan por sufrir las consecuencias sin conocer sus causas.

En la tabla 3 se observa que la ceguera es la incapacidad que se presenta con mayor intensidad en todos los estilos de aprendizaje del sector estudiado, por lo que podemos concluir que la deficiencia de no evaluar correctamente las amenazas y oportunidades del entorno es el problema más fuerte que sufre la industria en cuestión; además, independientemente de que este sea un sector competitivo, dejan de aprovecharse innumerables oportunidades.

En otras palabras, si los miembros del sector estudiado llegan a ser conscientes de las deficiencias de aprendizaje organizacional que padecen y trabajan al menos un poco en superarlas, se posicionarían en ventaja competitiva con relación a sus competidores.

En consecuencia, es imperativo que los gerentes y directivos de dichas empresas conozcan las causas de las deficiencias organizacionales que les aquejan para que las puedan superar y lleguen a ser más competitivos, sobre todo ahora que nuestro país necesita una industria más eficaz y competitiva.

Las dificultades que el presente estudio encontró consistieron sobre todo en una colaboración pobre por parte de los encuestados, así como su renuencia a proporcionar datos, que en su percepción consideraban poco importantes ya que aparentemente de manera directa no se reflejan en los índices de productividad y rentabilidad.

Por lo pronto, quedan algunas preguntas sin responder aunque pueden ser objeto de análisis en estudios posteriores; por ejemplo, la forma como se relaciona la cultura organizacional con los estilos de aprendizaje o con las incapacidades de aprendizaje, tópicos que pueden contribuir de manera más completa y profunda a explicar las variables de estudio, o inclusive vincular a estas 
con otras, tales como la supervivencia o tamaño de las empresas, o las estrategias de comercialización.

La aportación teórica más relevante del presente trabajo es la verificación de la influencia de las incapacidades en los estilos de aprendizaje, confirmando el estudio realizado por Yeung et al. (2000), y constituyendo un fundamento sólido para la toma de decisiones en las empresas.

\section{Bibliografía}

Argyris, C. (1999). Sobre el aprendizaje organizacional. México: Oxford University Press.

Barraza-Macías, A. (2007). ¿Cómo valorar un coeficiente de confiabilidad? Investigación educativa Duranguense (6): 6-10.

Beazley, H., Boenisch, J., \& Harden, D. (2003). Knowledge continuity: the new Management function. Journal of Organizational Excellence, 22(3), 65-81.

Cámara del Papel (2013), recuperado de: http://www.camaradelpapel.mx/trabajos-de-laseccion-tecnica/

Choo Wei, C. (1999). La Organización Inteligente: El Empleo de la Información para dar Significado, Crear Conocimiento y Tomar Decisiones. México: Oxford University Press.

De la Madrid, E. (2011) La situación de la industria de la celulosa y el papel en el mundo, publicaciones de Financiera Rural, recuperado de http://www.financierarural.gob.mx/informacionsectorrural/Documents/Articulos\%20F R/Microsoft\%20Word\%20\%20art\%C3\%ADculo\%20Celulosa\%20y\%20Papel.pdf

Etkin, J. (2007). La capacidad de gobernar en organizaciones complejas. Los acuerdos, la tensión creativa y tolerancia a la diversidad. Cali: Ed. Universidad del Valle.

Gómez-Romero, J.G.I. (2008). La Generación de Conocimiento Organizacional en la Micro, Pequeña y Mediana Empresas (Mipymes) de Durango. Durango: Editorial UJED. 
Gómez-Romero, J.G.I., Villarreal-Solís, F.M. \& Villarreal-Solís, M.D. (2014), Las incapacidades de aprendizaje organizacional y su relación con los estilos de aprendizaje organizacional en la MIPYME de la ciudad de Durango. Contaduría y administración, articulo en prensa.

Hair, J. F., Tatham, R. L., Anderson, R. E., \& Black, W. (2006). Multivariate data analysis (Vol. 6). Upper Saddle River, NJ: Pearson Prentice Hall.

Hernández, R, Fernández, C. \& Baptista, P., (2006), Metodología de la Investigación, México: McGraw Hill.

Hernández-Silva, F.\& Martí-Lahera, Y. (2006).Conocimiento organizacional: la gestión de los recursos y el capital humano. México: ACIMED

Huntington, E. (2001). La Tercer Vía, México: Editorial Diana.

Lind, D. A., Marchal, W. G., \& Wathen, S. A. (2005), Statistical techniques in business and economics, Irwin: McGraw Hill.

Nonaka, I. y Takeuchi, H. (2000). La Organización Creadora de Conocimiento: Cómo las Compañías Japonesas Crean la Dinámica de la Innovación. México: Oxford University Press.

Price Waterhouse Coopers (2011). Global Forest, Paper \& Packaging Industry Survey 2011 edition - survey of 2010 results, recuperado de www.pwc.com/fpp

Probst, G., Raub, S. \& Romhardt, K. (2001). Administre el conocimiento: los pilares del éxito, México: Prentice Hall.

Puerto-Becerra, P. (2010). La globalización y el crecimiento empresarial a través de estrategias de internacionalización. Pensamiento \& Gestión, 28,171-195.

Senge, P. (1993). La Quinta Disciplina. México: Ed. Granica.

Toffler, A. (1999). El Cambio del Poder. Barcelona: Plaza \& Janes Editores. 
Valdés, L. (2002). La Re-evolución Empresarial del Siglo XXI. Conocimiento y Capital Intelectual: Las Nuevas Ventajas Competitivas de la Empresa. Bogotá: Grupo Editorial Norma.

Watkins, K.E. \& Marsick, V.J. (1993).Sculpting the learning organization. New York: Jossey Bass.

Yeung, A., Ulrich, D., Nason, S. y Von Glinow, M. A. (2000). Las Capacidades del Aprendizaje en la Organización: Cómo Aprender a Generar Ideas con Impacto. México: Oxford University Press. 